El siguiente caso es de una EIRL que debiendo tributar en el régimen semi integrado, porque en forma habitual hace retiro sólo de la mitad de las utilidades, reinvirtiendo la otra mitad en su empresa queda por defecto o escoge erróneamente el régimen atribuido.

Igual que en el caso anterior, analicemos cuatro escenarios.

\begin{tabular}{|c|c|c|c|c|c|c|c|c|}
\hline Detalle & \multicolumn{2}{|c|}{ Renta Empresa M\$ 10.000} & \multicolumn{2}{|c|}{ Renta Empresa M\$ 50.000} & \multicolumn{2}{|c|}{ Renta Empresa M\$ 100.000} & \multicolumn{2}{|c|}{ Renta Empresa M\$ 5 50.00 } \\
\hline $\begin{array}{l}\text { Régimen } \\
\text { Tasa de Impuesto de Primera Categoría }\end{array}$ & $\begin{array}{c}\text { Régimen } \\
\text { Atribuidd } \\
25 \%\end{array}$ & $\begin{array}{l}\text { Régimen } \\
5-1 \\
27 \%\end{array}$ & $\begin{array}{l}\text { Résimen } \\
\text { Atribuido } \\
25 \%\end{array}$ & $\begin{array}{l}\text { Régimen } \\
s-1 \\
27 \%\end{array}$ & $\begin{array}{l}\text { Régimen } \\
\text { Atribuido } \\
\text { 25\% }\end{array}$ & $\begin{array}{l}\text { Régimen } \\
5-1 \\
27 \%\end{array}$ & $\begin{array}{l}\begin{array}{c}\text { Régimen } \\
\text { Atribuido } \\
\text { 25\% }\end{array} \\
\text { 25\% }\end{array}$ & $\begin{array}{c}\text { Régimen } \\
\text { S-1 } \\
27 \%\end{array}$ \\
\hline $\begin{array}{l}\text { Renta Liquida Imponible } \\
\text { impuesto Renta Primera Categoría (IDPC) }\end{array}$ & $\begin{array}{l}10.000 \\
2.500\end{array}$ & $\begin{array}{l}10.000 \\
2.700\end{array}$ & $\begin{array}{l}50.000 \\
12.500\end{array}$ & $\begin{array}{l}50.000 \\
13.500\end{array}$ & $\begin{array}{l}100.000 \\
25.000\end{array}$ & $\begin{array}{l}100.000 \\
27.000\end{array}$ & $\begin{array}{l}500.000 \\
125.000\end{array}$ & $\begin{array}{l}500.000 \\
135.000\end{array}$ \\
\hline $\begin{array}{l}\text { Base Impuesto GC - atribución } \\
\text { Base Impuesto GC - retiro } 50 \% \text { utilidades }\end{array}$ & 10.000 & 5.000 & 50.000 & 25.000 & 100.000 & 50.000 & 500.000 & 250.000 \\
\hline $\begin{array}{l}\text { obal Complementario } \\
\text { IDPC }\end{array}$ & $\begin{array}{l}109 \\
2.500\end{array}$ & $\begin{array}{c}0 \\
1755\end{array}$ & $\begin{array}{r}5.598 \\
1.2500\end{array}$ & $\begin{array}{l}1.061 \\
8.775\end{array}$ & $\begin{array}{l}23.455 \\
25.000\end{array}$ & $\begin{array}{c}5.598 \\
17.50\end{array}$ & $\begin{array}{l}183.455 \\
125000\end{array}$ & $\begin{array}{l}83.455 \\
8750\end{array}$ \\
\hline 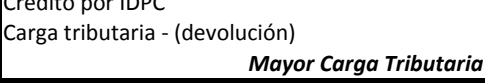 & $\begin{array}{l}2.500 \\
-2.391\end{array}$ & $\begin{array}{l}1.1755 \\
-1.755 \\
636\end{array}$ & $\begin{array}{l}\frac{12.300}{-6.902} \\
811\end{array}$ & $\begin{array}{l}8.775 \\
-7.714\end{array}$ & $\begin{array}{l}2.0 .00 \\
-1.54 \\
10.407 \\
\end{array}$ & 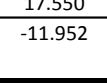 & $\begin{array}{l}158.000 \\
58.75 \\
62.750\end{array}$ & 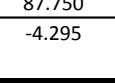 \\
\hline
\end{tabular}

\section{Oe}

- El primer escenario de renta empresa $\mathrm{M} \$ 10.000$ y hasta $\mathrm{M} \$$ mentadas, no es dificil prever -cuestión que ya se comenta en 43.900, al propietario efectivamente convendría el régimen atri- distintos circulos- que durante una próxima administración, verá buido, bajo los supuestos de retiros planteados, ya que desde la la luz una nueva reforma tributaria que -ojala- se desarrolle lejos renta de M\$ $\$ 3.900$ hacia arriba, provocaria exceso indebido de de la imaginación surrealista de sus creadores y se abstraiga de tributación.

- En los siguientes tres escenarios, bajo el mismo análisis, el indebido exceso de carga tributaria alcanza la suma de $\mathrm{M} \$ 811$ M\$ 10.407 y M\$ 62.750 respectivamente. especialistas en el área y se ciña alrededor de los deseables atributos anteriormente comentados.

Mientras tanto, una ley transitoria podria establecer un periodo de marcha blanca para ayudar a contribuyentes (sobre todo Como podemos apreciar en estos simples ejemplos, la imposi- a péqueñas y medianas empresas) a retractarse y modificar su ción de un determinado régimen de tributación o la elección erratributaria, de su incapacidad financiera de recurrir a la asesoria tel conocimiento experto o de eventuales cambios futuros en su estructura de retiros de utilidades desde su empresa, pueden provocar importantes detrimentos patrimoniales en un contribuvente, obligándolos además, a permanecer coactivamente por cinco años en ese indeseado régimen

En términos generales, si interpelamos al sentido común y hacemos eco de la opinión de ex ministros, autoridades y de especialistas en tributación sobre la incertidumbre en la certeza juridica de las actuales y futuras interpretaciones legales en esta materia y si además, obviamos el sesgo ideológico que en parte motivó aspectos importantes de la actual reforma y con-
sideramos las dificultades de implementación de las normas coario? Lo dudo.

'Documento del SII: "Diagnóstico del Sistema Tributario Chileno", 2016, http:ll

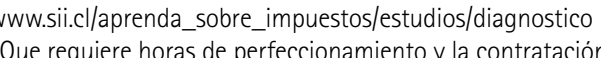

ación de servicios tribuarios de apoyo, no considerados en el presupuesto de su gestión. incrementos en niveles de cappacitación de fiscalizadores $y$ i ari en importantes Iscales "Compartiendo, entre otras propuestas, I la del director de Clapes UC: "... volver solo régimen, eliminando la renta atribuida..." El Mercurio 5/8/2016

\title{
Incentivos tributarios a las donaciones
}

\author{
Andrea Butelmann, Ph. D Economia, Universidad de \\ Chicago y directora del Magíster en Economía Aplicada
} a Politicas Públicas (MAPE),UAH.

Pedro Rojo, egresado del Programa de Magister en Economia

Durante el vittimo tiempo en nuestro pais, producto de diversos desastres naturales se ha puesto de manifiesto el rol de las empresas, fundaciones y grandes patrimonios personales respecto Andrónico Luksic, Leonardo Farkas y otras personas que anónimamente realizan aportes para efectos de colaborar con una primera trofes generadas por lo naturaleza, motivan a reflexionar respecto de si en nuestro pais -con independencia de las circunstancias particulares que conllevan a realizar donaciones millonariasexisten incentivos sociales, económicos, políticos o de otro orden para que la institución de la donación sea una práctica habitual y no un hecho aislado y/o circunstancial.

A finales del año 2016, el Centro de Filantropía e Inversiones Sociales (CEFIS) de la Universidad Adolfo lbáñez, publicó un mapeo de filantropia e inversiones sociales a nivel pais, cuyo objetivo principal fue indagar respecto de la percepción ciudadana' relacionada con las donaciones y contribuciones sociales de las empresas en Chile.

Del estudio publicado es posible converger en dos aspectos reevantes a considerar:

1. Tanto salud como educación (primaria) son señaladas como

haciones debieran ser reconocidos como gastos proptos por dozar de ningún tipo de beneficio tributario. teamiento, la respuesta inmediata de la mitad de los encuestado sin embargo es importante determinar el contexto adecuado de como opera la institución de la donación en Chile. En este caso, analizaremos el incentivo tributario de donaciones con fines educacionales en la educación superior (en general, las donaciones sociales, deportivas, entre otras permitidas por la Ley para acceder a beneficios tributarios operan de la misma forma). Esto se hace especialmente relevante dado que hay personas de alto patrmonio que si bien, efectuan donaciones a instituciones en Chile entender dadas las carencias locales.

\section{ASPECTOS GENERALES}

En términos generales, las donaciones entre vivos se encuentran reguladas en el Libro III, Titulo XIII del Código Civil, artículos 386 y siguientes. El concepto de donación, está definido como parte de sus bienes a otra persona, que la acepta" (artículo 138 del Código Civil)

El ordenamiento juridico chileno ha establecido beneficios tributarios relacionados con las donaciones con el objeto de fomentarlas. En relación a esto, se establecen en distintos cuerpos legales, las normas para acogerse a estos beneficios tributarios. Asi entonces y por regla general, los beneficios tributarios que stablecen las leyes en materia de donaciones, corresponden a as siguientes:

Exención del trámite de insinuación. ${ }^{2}$

3. Rebajar dichos conceptos como gastos necesarios para producir la renta lísuida imponible sujeta del impuesto de prime-

4. Otorgar créditos en el pago de determinados impuestos.

En lo que respecta de la exención del impuesto a las donaciones, este tiene por finalidad gravar la donación ${ }^{3}$ con una tasa de impuesto que fluctúa entre $1 \%$ a $25 \%$ por parte del donatario(quien recibe la donación), aplicando las mencionadas tasas impositivas sobre el valor monetario de la donación. Cabe precisar
Oe 
que la tasa de impuesto es progresiva, es decir, de acuerdo a tramos estructurados en UTM (se aplican deducciones fijas en cada uno de los tramos), comenzando desde 0,1 UTM a 14.440 UTM y más, se aplican las tasas de impuestos mencionada anteriorde relación familiar entre el donante y donatario, se aplica una sobretasa de $40 \%$

La tasa de impuestos a las donaciones es el primer incentivo a cios que se describen a continución. Sin embargo, esa posiblidad es bastante restringida. En efecto, respecto a los purtos 3 y 4 que se refieren a reducciones en el impuesto al ingreso podemos realizar un vinculo en la respuesta del 50\% de los encuestados del estudio de CEFIS que se oponen a que las empresas puedan utilizar algún beneficio tributario cuando realizan donaciones.

Lo anterior porque en lo particular no cualquier gasto puede ser deducible de la base imponible sobre la cual pagan impuestos las empresas (o bien las personas), esto dado que la misma Ley de Impuesto a la Renta (LIR) señala que los gastos deducibles de la base imponible, deben cumplir con ciertos requisitos. EI
más común de todos es que las erogaciones sean necesarias más común de todos es que las erogaciones sean necesarias

legislador cabe preguntarse i Por qué se debe punto de vista del sobre cual se aplica el impuesto que deben regar las empresas anualmente las donaciones que esta realiza? iTiene alguna correlación el gasto en que se incurre por dicho concepto con los ingresos que puedan generar? ¿Es una correcta asignación de recursos las donaciones en desmedro de la recaudación fiscal? En este sentido, para efectos de incentivar a que las donaciones efectuadas por las empresas sean consideradas como gastos para deducir la base imponible sobre la cual se aplican los impuestos, se han creado diversos mecanismos que también producen distorsiones o desincentivos.

ASPECTOS PARTICULARES RESPECTO DE DONACIONES A UNIVERSIDADES E INSTITUTO PROFESIONALES

Tal como se señaló, en el caso particular de las donaciones que se efectúan a Universidades o Institutos Profesionales,

mismas deben circunscribirse a los siguientes aspectos:

-Adquisicion de bienes inmuebles y de equipamiento, como apoyar el perfeccionamiento del quehacer académico.

-Proyectos de investigación emprendidos por las mencionadas instituciones.

En este contexto, los donatarios pueden ser aquellas empresas sujetas del Impuesto de Primera Categoría (IDPC) o en su defecto Personas Naturales, que realizando aportes solo en dinero Universidades e Instituto Profesionales Estatales y/o Particulares reconocidos por el Estado, gocen de ciertos beneficios y cumpla con requisitos y condiciones particulares
El beneficio particular consiste en un crédito contra el IDPC . considerando un Limite ( sobre cual se aplica el IDPC. El mencionado crédito opera en la el IDPC y por lo tanto se traduce en un beneficio de flujos directamente a la empresa que llevó a cabo la donación.

Sin embargo, se debe tener presente que este crédito no puede exceder en ningún caso el equivalente de 14.000 UTM (aproxño. El monto que se determine finalmente como credito se inputará al IDPC determinado para el año en que se efectuó donación. En caso que se genere un excedente respecto del IDPC, el remanente de crédito podrá imputarse en los siguientes años. A su vez, el 50\% restante de la donación corresponderá a ser deducibles de la base imponible del IDPC, siempre y cuando el mismo gasto no exceda el 2\% de dicha base o el 0,16\% del Capital Propio Tributario (determinación contable considerando las normas de la LRT).

Por otra parte, aquellas personas naturales que reciben sueldos, dividendos u otro tipo de ingresos, tambien pueden ser donantes y acogerse al beneficio tributario analizado, optando (IGC) el cual no puede ser superior al $20 \%$ de Complementario

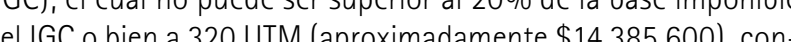
siderándose el limite menor. Sin embargo a diferencia del caso de las empresas, el 50\% restante de la donación simplemente no puede ser deducida de la base imponible del cálculo del IGC. De acuerdo a lo anterior, existe claramente un incentivo realizar donaciones desde una empresa en contraste de hacerlo como persona natural ya que el incentivo para las empresas es mayor, sin embargo respecto de la cuantia de los montos

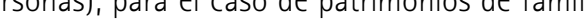
mportantes del pais, podrian parecer cifras menores, las cuain al exceder los maximos señalados se sujetan del pago de un . (Impuesto denominado "Gastos echazados")

Una forma de evitar el uso de empresas para hacer donaciones por parte de grandes patrimonios es a través de la creación de fundaciones. Esto permite realizar aportes por sobre los máximos b renta y y que las fundaciones no son sujetas de impuesto a quiere de la realización del trámite de insinuación requerido para las donaciones, en tanto tales aportes no se consideran donación sino una destinación de bienes.

Sin embargo, en muchas situaciones particulares las fundaciones son utilizadas para fines accesorios, principalmente para diferir el impuesto a las herencias y de sucesión, resguardar patrimonios en el exterior, entre otras razones.

. into el caso de Estados Unidos en

\begin{tabular}{|c|c|c|c|c|c|}
\hline DETALLE & $\begin{array}{l}\text { AÑO } \\
\text { TRIBUTARIO } \\
2012\end{array}$ & $\begin{array}{l}\text { AÑ̃ } \\
\text { TRIBUTARIO } \\
\text { 2013 }\end{array}$ & $\begin{array}{l}\text { AÑ̃ } \\
\text { TRIBUTARIO } \\
2014 \\
\end{array}$ & $\begin{array}{l}\text { AÑ̃O } \\
\text { TRIBUTARIO } \\
2015\end{array}$ & $\begin{array}{l}\text { AÑO } \\
\text { TRIBUTARIO } \\
\text { 2016 }\end{array}$ \\
\hline Número de donatarios & 50 & 52 & 50 & 53 & 50 \\
\hline Vúmero de donantes & 881 & 822 & 846 & 830 & 763 \\
\hline $\begin{array}{l}\text { Monto anual de donaciones informado } \\
{[M M \$ \text { del año] }}\end{array}$ & $25.018,41$ & $23.531,62$ & $24.627,22$ & $22.410,72$ & $28.962,77$ \\
\hline $\begin{array}{l}\text { Crédito al IGC por donaciones a Universidades e } \\
\text { nstitutos Profesionales (Art.69 Ley No18.681/87) [MM \$ } \\
\text { del año] }\end{array}$ & 138 & 247 & 170 & 140 & 122 \\
\hline $\begin{array}{l}\text { Crédito por donaciones Universidades e } \\
\text { nst.Profesionales [MM } \$ \text { del año] }\end{array}$ & 318 & 324 & 338 & 265 & 250 \\
\hline
\end{tabular}

que las personas pueden deducir por efecto de donaciones hasta el 50\% de su ingreso, sin limites de montos de su base tributaria. Por otra parte, de acuerdo a la información proporcionada no Transparente, los montos informados como donaciones so relativamente bajos, comparando nivel de donación y el monto máximo de crédito que es posible deducir por las empresas. Sólo a modo de ejemplo, para el año tributario 2016, el monto anua de donaciones informadas, equivale al 0,26\% de la capitalización bursátil de una de las companias chilena como Falabella, de mayor presencia bursatil. (MM\$ 10.994.044)

Como se ha expuesto, existen diversos incentivos para llevar a ficios que otorgan a los donantes, todavia son restrictivas yanelimitan los montos que pueden ser utilizados como por ejemplo en la deducción de la base imponible sujetas de impuestos, o en otorgamiento de un crédito contra el pago del mismo. Pese a que

los montos involucrados son relativamente bajos, se debe cumplir con un sin número de verificaciones de limites sobre los cuales se generan beneficios, además de información que se entrega a a autoridad fiscal tanto del donante como del donatario lo que tiene como consecuencia que finalmente, empresas y personas, hivos a intervenir (como se hace en otros paises) al desarrollo $d$ de pais A estos limites se se hace en otros palses) al desartiva en as dirigencias estudiantiles a valorar tales donaciones, que pued estar funcionando como disuasivo para considerar donar a universidades.

por dan de la discusión si se deben dar o no rebajas tributarias quisieramos tener para quado democracia tributaria que en qué se gasta los tributos al ingreso impuestos por el Estado). los autores no ven la lógica de desincentivar la filantropía con el impuesto a las donaciones y la sobre tasa correspondiente.

TEl universo de la encuesta correspondió a chilenos, hombres y mujeres
mayores de 18 años habitantes de las 73 comunas urbanas con äs de 50 personas que representan el $70.9 \%$ del total del pais. "Definido como "la autorización de juez competente, solicitada por el donante del Código de Procedimiento Civill,

Para efectos de determinar la base imponible, solo en los casos en que el
donatatrio es cónyuge del donatario, o bien los ascendientes o descendientes el donatario, se debe proceder a rebajar 60 UTM según valor a la fecha de la

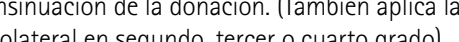
"Articulo 69 de la Ley No $18.681 / 87$ y
DS de Hacienda No 340, de 1988. EEl Limite General Absoluto (LGA) regulado por el Articulo 10 de la Ley N 19.885 del año 2003 , consiste en determinar un tope total de donaciones
(Donaciones por otros conceptos definidos por leyes distintas l a analizadd) efectuadas en un año calendario.
Fono Facultad: 28897366 e-mail: jrodrigu@uahurtado.c

fen.uahurtado.cl
Oe 